\title{
Thrombocytopenia After Transcatheter Valve- in-Valve Implantation: Prognostic Marker or Mere Finding?
}

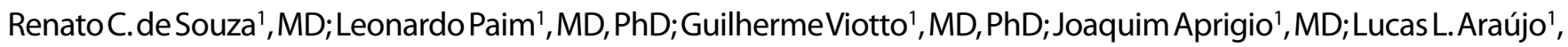
MD; Henrique Ribeiro', MD; Roney O. Sampaio', MD, PhD; Flavio Tarasoutchi', MD, PhD; Pablo M. A. Pomerantzeff', MD, PhD; José Honório Palma' ${ }^{1}, \mathrm{MD}$, PhD; Fabio B. Jatene', $\mathrm{MD}$, PhD

DOI: 10.21470/1678-9741-2018-0078

\section{Abstract}

Objective: To analyze the behavior of platelets after transcatheter valve-in-valve implantation for the treatment of degenerated bioprosthesis and how they correlate with adverse events upon follow-up.

Methods: Retrospective analysis of 28 patients who received a valve-in-valve implant, 5 in aortic, 18 in mitral and 5 in tricuspid positions. Data were compared with 74 patients submitted to conventional redo valvular replacements during the same period, and both groups' platelet curves were analyzed. Statistical analysis was conducted using the IBM SPSS Statistics $₫ 20$ for Windows.

Results: All patients in the valve-in-valve group developed thrombocytopenia, $25 \%$ presenting mild $(<150.000 / \mu \mathrm{L})$, $54 \%$ moderate $(<100.000 / \mu \mathrm{L})$ and $21 \%$ severe $(<50.000 / \mu \mathrm{L})$ thrombocytopenia. The platelet nadir was on the $4^{\text {th }}$ postoperative day for aortic ViV, $2^{\text {nd }}$ for mitral and $3^{\text {rd }}$ for tricuspid patients, with the majority of patients recovering regular platelet count. However, the aortic subgroup comparison between valve-invalve and conventional surgery showed a statistically significant difference from the $7^{\text {th }}$ day onwards, where valve-in-valve patients had more severe and longer lasting thrombocytopenia. This, however, did not translate into a higher postoperative risk. In our study population, postoperative thrombocytopenia did not correlate with greater occurrence of adverse outcomes and only normal preoperative platelet count could significantly predict a postoperative drop $>50 \%$.

Conclusion: Although thrombocytopenia is an extremely common finding after valve-in-valve procedures, the degree of platelet count drop did not correlate with greater incidence of postoperative adverse outcomes in our study population.

Keywords: Thrombocytopenia. Transcatheter Aortic Valve Replacement. Heart Valve Prosthesis Implantation. Heart Valves/ Surgery.

\begin{tabular}{ll}
\hline \multicolumn{2}{l}{ Abbreviations, acronyms \& symbols } \\
\hline AKI & $=$ Acute kidney injury \\
BAV & $=$ Balloon aortic valvuloplasties \\
CABG & $=$ Coronary artery bypass grafting \\
CPB & $=$ Cardiopulmonary bypass \\
HIT & $=$ Heparin-induced thrombocytopenia \\
IABP & $=$ Intra-aortic balloon pumps \\
ICU & $=$ Intensive care unit \\
LODS & $=$ Logistic Organ Dysfunction Score
\end{tabular}

${ }^{1}$ Cardiovascular Surgery Division, Instituto do Coração do Hospital das Clínicas da Faculdade de Medicina da Universidade de São Paulo (InCor-HCFMUSP), São Paulo, SP, Brazil.

This study was carried out at Cardiovascular Surgery Division, Instituto do Coração do Hospital das Clínicas da Faculdade de Medicina da Universidade de São Paulo (InCor-HCFMUSP), São Paulo, SP, Brazil.

Financial Support: Partial external financial support was received for the development of this study, in the form of donations of transcatheter heart valves performed by Braile Biomédica.
Conflict of Interest: The authors would like to declare that the institution has received research grants from Braile Biomédica.

\section{Correspondence Address:}

Leonardo Paim Nicolau da Costa

Av. Dr. Enéas de Carvalho Aguiar, 44 - São Paulo, SP, Brazil

Zip code: 05403-900

E-mail: drleonardopaim@gmail.com 


\section{INTRODUCTION}

Thrombocytopenia is defined as an absolute platelet count $<150.000 / \mu \mathrm{L}$ or a decrement greater than $50 \%$ from baseline value ${ }^{[1]}$. This is a common finding in critically ill patients classically associated with poor prognosis, prolonged hospital stay, higher risk and, ultimately, reduced survival[2]. Consequently, platelet count has been implicated as a prognostic marker for various severity scoring systems, such as Multiple Organ Dysfunction Score $(\mathrm{MODS})^{[3,4]}$, Sequential Organ Failure Assessment (SOFA) ${ }^{[5]}$, Logistic Organ Dysfunction Score (LODS) ${ }^{[6]}$, and others ${ }^{[7]}$.

There are basically four mechanisms that can decrease platelet counts: decreased production, increased destruction (immuneor nonimmune-mediated), hemodilution and sequestration (as in hypersplenism) ${ }^{[1,2,8,9]}$. In the general intensive care unit (ICU) scenario, thrombocytopenia may occur in up to $20 \%$ admissions, $35 \%$ of surgical hospitalizations and $45 \%$ of trauma admissions ${ }^{[1]}$. Considering cardiac surgery patients, thrombocytopenia is an extremely frequent finding, varying from $35 \%$ to $65 \%$ of all cases $^{[10]}$, and patients after percutaneous coronary interventions $(\mathrm{PCl})$ are also prone to develop major thrombocytopenia, i.e. $<100.000 / \mu \mathrm{L}$, in $2.4-9.2 \%$ of cases $^{[11-13]}$.

Causes for thrombocytopenia following cardiac surgery are believed to be multifactorial, with cardiopulmonary bypass (CPB) installation as the main culprit. However, with the development of off-pump coronary artery bypass grafting (CABG) and more recent transcatheter valve procedures, such as transcatheter aortic valve replacement (TAVR), new questions arose regarding thrombocytopenia in a post-cardiac surgery setting. Even without the use of CPB, platelet count continues to drop, which rendered further doubts not only as to the origin of this problem, but also to its clinical implications. To date, there are very few studies available which tackle this issue, with some showing strong correlation between the occurrence of severe thrombocytopenia after TAVR and higher mortality rates ${ }^{[14]}$.

When we consider the valve-in-valve (ViV) scenario and the incidence of thrombocytopenia, even less can be said. Although transcatheter ViV procedures is a well-established option for treatment of degenerated bioprosthesis in high and extreme-risk patients ${ }^{[15]}$, and the incidence of thrombocytopenia is high after these procedures, there are no available data in the literature addressing this problem. Therefore, little is known about this phenomenon and its correlation with unfavorable events.

\section{METHODS}

We carried on a retrospective, single-centre, database analysis study of all consecutive patients who underwent transcatheter ViV implantation from May 2015 until March 2017. A total of 30 patients underwent transcatheter ViV procedures at our institution during this period, with the majority of them performed through transapical access (82\%). One patient who had transcatheter mitral ViV and concomitant TAVR, and one patient who received two concomitant ViVs for mitral and tricuspid valve degeneration, were not included in this study, leaving the remainder of 28 patients. Of these, 5 were performed on degenerated bioprosthetic valves on aortic position, 18 on mitral and 5 on tricuspid. Four of the five patients in the tricuspid group had transjugular access, and one patient was accessed through a transatrial right anterior thoracotomy. All patients who underwent ViV procedures were deemed high or extreme risk for conventional approach, and were thoroughly discussed by our heart team. Surgical procedures were conducted in a hybrid operating room, using fluoroscopic and echocardiographic guidance. The transcatheter valve utilized in all patients was the Braile Inovare prosthesis, a balloon expandable valve platform with a chromium-cobalt stent frame, widely used in Brazilian experience ${ }^{[16,17]}$.

Pre- and postoperative data were collected and the platelet curves were elaborated, starting with the first value upon ICU admission, and taking into account daily values of platelet counts until the $10^{\text {th }}$ postoperative day. Postoperative bleeding was also quantified and correlated with the drop in platelet values, as well as the occurrence of other adverse events, such as paravalvular regurgitation, acute renal failure and death. All definitions were in accordance with the Valve Academic Research Consortium (VARC-2) standardized endpoint definitions for TAVR consensus document ${ }^{[18]}$.

Furthermore, data was collected from all conventional reoperations for degenerated bioprosthetic valve replacement, performed at our institution during the same period to serve as a control group. A total of 146 patients were analyzed and 74 were included, after patients with active infective endocarditis, combined procedures (i.e.: CABG + valve replacement), previous valve repair and non-replacement, use of mechanical valves at any point, septic shock, previous hematologic disease, pregnant women and those with perioperative mortality were excluded. Postoperative platelet curves between both groups were compared and correlated with clinical outcomes.

\section{Statistical Analysis}

For the variables with homogeneous distribution, parametric tests were performed and the results were presented in mean and standard deviation. As for the variables with non-homogeneous distribution, we performed non-parametric tests and the results are presented in median and interquartile range. Chi-square test and Fisher's exact test were used to compare the categorical variables. Analysis of the quantitative variables was performed by comparing means using the t-test or Kruskal-Wallis test. We considered as statistically significant differences the results with values of $P<0.05$. Statistical analysis were conducted using IBM SPSS Statistics 20 for Windows (Chicago, IL, United States).

\section{RESULTS}

Thrombocytopenia occurred in 100\% of patients who underwent transcatheter ViV implantation, regardless of the position where the degenerated bioprosthesis was originally placed.Seven (25\%) patients experienced mild thrombocytopenia, with the nadir of platelet counts between 100.000-150.000/ $\mu \mathrm{L}$, 15 (54\%) showed moderate thrombocytopenia (50.000-100.000/ $\mu \mathrm{L})$ and $6(21 \%)$ presented with severe thrombocytopenia postoperatively $(<50.000 / \mu \mathrm{L})$, as shown in Table 1 . Within this group, there was no statistical difference between the degree of thrombocytopenia presented and the occurrence of adverse events, such as death, major bleeding, acute renal failure and 
Table 1. Baseline and postoperative characteristics of valve-in-valve patients according to the degree of thrombocytopenia, in absolute values.

\begin{tabular}{|c|c|c|c|c|c|}
\hline & General & \begin{tabular}{|c|} 
Mild \\
thrombocytopenia \\
$(\geq 100.000 / \mu \mathrm{L})$ \\
7 patients
\end{tabular} & $\begin{array}{c}\text { Moderate } \\
\text { thrombocytopenia } \\
\text { (<100.000/ } \mu \mathrm{L}) \\
15 \text { patients }\end{array}$ & $\begin{array}{c}\text { Severe } \\
\text { thrombocytopenia } \\
\text { ( } \leq 50.000 / \mu \mathrm{L}) \\
6 \text { patients }\end{array}$ & $P$-value \\
\hline \multicolumn{6}{|l|}{ Preoperative } \\
\hline Age & $60(16-82)$ & $59(16-80)$ & $60(34-81)$ & $62(21-78)$ & 0.796 \\
\hline EuroSCORE II & $7.45(2,86-32.87)$ & $9.85(1.52-32.93)$ & $8.75(2.52-26.87)$ & $10,10(1.52-26.46)$ & 0.943 \\
\hline NYHA I-II & $9(32.14)$ & $3(42.85)$ & $4(26.6)$ & $2(40)$ & 0.753 \\
\hline NYHA III-IV & $19(67.85)$ & $4(57.14)$ & $11(73.3)$ & $3(60)$ & 0.753 \\
\hline Chronic renal failure & $15(53.57)$ & $4(57.14)$ & $8(53.3)$ & $3(50)$ & 0.967 \\
\hline Atrial fibrillation/flutter & $17(60.71)$ & $5(71.4)$ & $10(66.6)$ & $2(33.3)$ & 0.3 \\
\hline CAD & $10(35.71)$ & $3(42.8)$ & $4(26.6)$ & $3(50)$ & 0.543 \\
\hline DM & $7(25)$ & $2(28.6)$ & $4(26.6)$ & $1(16.6)$ & 0.856 \\
\hline CABG & $7(25)$ & $2(28.6)$ & $4(26.6)$ & $1(16.6)$ & 0.856 \\
\hline Previous prosthesis stenosis & $12(43)$ & $2(29)$ & $4(40)$ & $4(67)$ & 0.361 \\
\hline Previous prosthesis insufficiency & $16(57)$ & $5(71)$ & $9(60)$ & $2(33)$ & 0.361 \\
\hline No preoperative thrombocytopenia & $16(57.14)$ & $5(71.4)$ & $8(53.3)$ & $3(50)$ & 0.663 \\
\hline Mild preoperative thrombocytopenia & $8(28.6)$ & $2(28.6)$ & $5(33.3)$ & $1(16.6)$ & 0.732 \\
\hline Moderate preoperative thrombocytopenia & $4(14.28)$ & $0(0)$ & $2(13.3)$ & $2(33.3)$ & 0.17 \\
\hline \multicolumn{6}{|l|}{ Postoperative } \\
\hline Absent or mild paravalvular regurgitation & $25(92)$ & $6(85.7)$ & $15(100)$ & $4(80)$ & 0.071 \\
\hline Moderate paravalvular regurgitation & $2(7.4)$ & $1(14.2)$ & $0(0)$ & $1(20)$ & 0.071 \\
\hline Death & $5(17.8)$ & $1(14.28)$ & $2(13.33)$ & $2(33.3)$ & 0.572 \\
\hline Major bleeding & $2(7.1)$ & $1(14.3)$ & $0(0)$ & $1(16.7)$ & 0.196 \\
\hline Acute renal failure & $17(60.7)$ & $3(42.8)$ & $10(66.6)$ & $4(66.6)$ & 0.542 \\
\hline Sepsis & $11(39.28)$ & $2(28.6)$ & $7(46.6)$ & $2(33.3)$ & 0.677 \\
\hline
\end{tabular}

$\mathrm{CABG}=$ coronary artery bypass grafting; $\mathrm{CAD}=$ coronary artery disease; $\mathrm{DM}=$ diabetes mellitus; $\mathrm{NYHA}=$ New York Heart Association

sepsis. Regarding baseline characteristics, there was also no significant factor that could predict a higher risk of postoperative thrombocytopenia.

\section{Platelet Curves for ViV Patients}

In every position where the transcatheter valve was implanted, patients presented with a drop in platelet counts already at the immediate postoperative period, as shown in Figure 1.

Of note, four out of five patients who had a ViV implanted in tricuspid position were preoperatively thrombocytopenic (average preoperative platelet count of $121.600 / \mu \mathrm{L}$ ), two with mild and two with moderate thrombocytopenia. The nadir of the tricuspid curve occurred at the $3^{\text {rd }}$ postoperative day, and by the $10^{\text {th }}$ day, the mean platelet count had reached preoperative values, but remained below the normal threshold of 150.000/ $\mu \mathrm{L}$. In this subgroup of patients, the nadir of the platelet curve represented a $36.8 \%$ drop from baseline.
In the mitral ViV subgroup, the preoperative platelet count was normal, with an average of $180.278 / \mu \mathrm{L}$. Thrombocytopenia was observed in the immediate postoperative period, with the nadir occurring at the $2^{\text {nd }}$ postoperative day. Platelet counts began to rise from the $3^{\text {rd }}$ day onward, reaching normal values at the $7^{\text {th }}$ postoperative day. In turn, the nadir of the curve represented a $45.3 \%$ fall in relation to preoperative values.

Aortic ViV patients also presented with normal preoperative platelet counts, and had the highest values amongst the three subgroups: $198.400 / \mu \mathrm{L}$. These patients also experienced a platelet drop in the immediate postoperative period; however, thrombocytopenic values were only reached at the $1^{\text {st }}$ postoperative day and persisted for longer. The nadir of the curve occurred in the $4^{\text {th }}$ day and, until the $10^{\text {th }}$ day, regular preoperative levels had not yet been reached, unlike the other two subgroups. The aortic ViV subgroup of patients presented with the most expressive drop in platelet count: $62.3 \%$.

Despite differences in absolute and percentagevalues between 


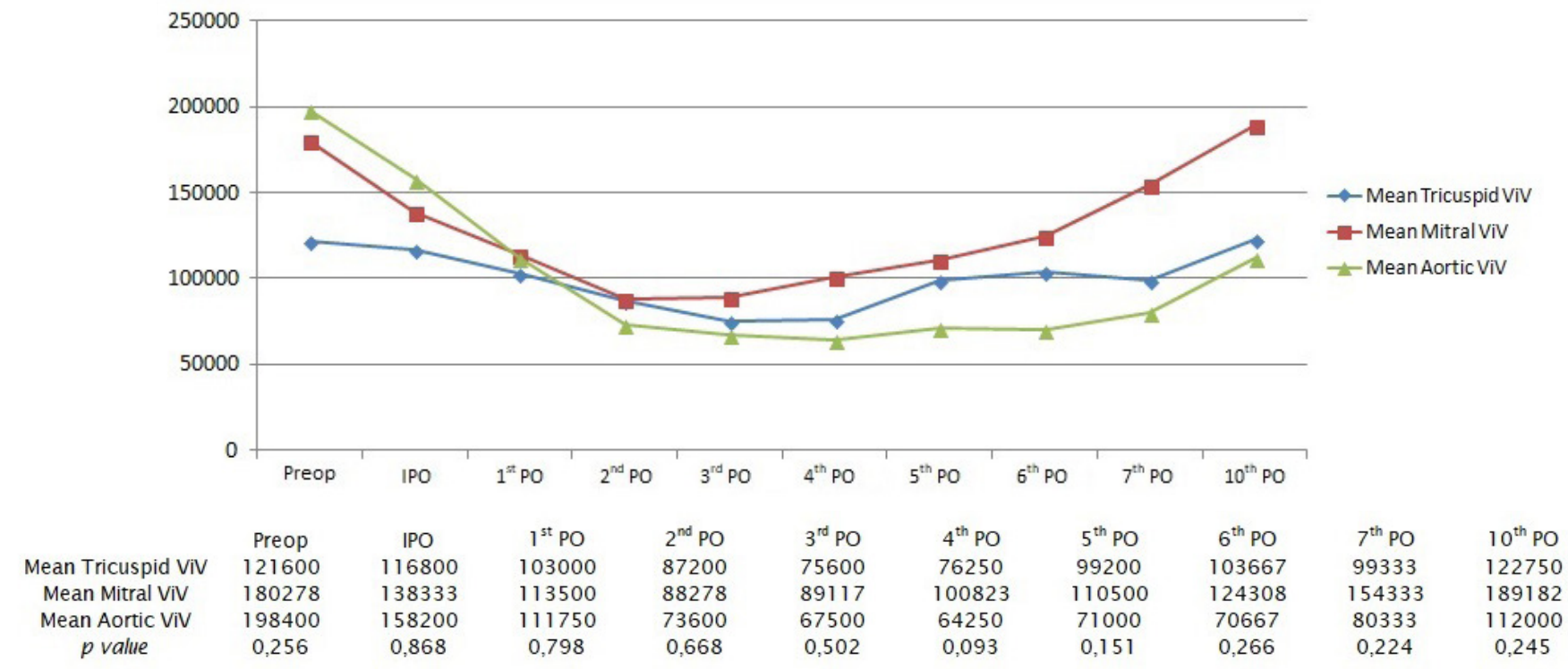

Fig. 1 - Mean platelet curves for all patients who underwent a valve-in-valve procedure. $I P O=$ immediate postoperative period; $P O=$ postoperative day; ViV=valve-in-valve

the three subgroups, they were not statistically significant.

\section{Platelet Curves for ViV vs. Conventional Reoperations}

The control group consisted of 74 patients who underwent conventional reoperations to replace degenerated bioprosthesis at our institution, during the same period. Of these, 31 were submitted to redo aortic valve replacement and 43 to redo mitral valve replacement. No isolated tricuspid redo patients could be included because these were all congenital heart disease patients, who usually underwent combined procedures, or had other complex cardiac repairs previously done, which frequently utilized synthetic material and had to be excluded.

The comparison between patient's preoperative characteristics in each group is shown in Tables 2 and 3. As expected, ViV patients were older and had more comorbidities, such as chronic renal failure, coronary artery disease and previous CABG. These differences were statistically significant and became more evident in the mitral group comparison. Although EuroSCORE II was higher in both ViV subgroups, this aspect showed no statistical difference.

The comparison between conventional mitral valve reoperation and mitral ViV showed very similar platelet curves, as shown in Figure 2. Thrombocytopenia was present at the

Table 2. Preoperative characteristics comparison between 18 mitral ViV patients and 43 conventional mitral reoperations.

\begin{tabular}{l|c|c|c}
\hline & Mitral ViV & $\begin{array}{c}\text { Mitral conventional } \\
\text { reoperation }\end{array}$ & $\boldsymbol{P}$-value \\
\hline Age & 63.9 & 54.7 & 0.025 \\
\hline EuroSCORE II & $11.48 \%$ & $7.59 \%$ & 10.075 \\
\hline NYHA I-II & $4(22.2)$ & $33(76.7)$ & 1 \\
\hline NYHA III-IV & $14(77.8)$ & $13(30.23)$ & 0.011 \\
\hline Chronic renal failure & $12(66.7)$ & $26(60.5)$ & 0.775 \\
\hline Atrial fibrillation/flutter & $12(66.7)$ & $4(9.3)$ & 0.051 \\
\hline CAD & $6(33.3)$ & $4(9.3)$ & 0.108 \\
\hline DM & $5(27.8)$ & $1(2.3)$ & 0.024 \\
\hline Previous CABG & $4(22.2)$ & $29(67.4)$ & 0.022 \\
\hline Previous prosthesis insufficiency & $6(33.3)$ & $31(72.1)$ & 0.547 \\
\hline
\end{tabular}

$\mathrm{CABG}=$ coronary artery bypass grafting; $\mathrm{CAD}=$ coronary artery disease; $\mathrm{DM}=$ diabetes mellitus; NYHA=New York Heart Association 
Table 3. Preoperative characteristics comparison between 5 aortic ViV patients and 31 conventional aortic reoperations.

\begin{tabular}{l|c|c|c}
\hline & Aortic ViV & $\begin{array}{c}\text { Aortic conventional } \\
\text { reoperation }\end{array}$ & P-value \\
\hline Age & 79 & 61.8 & $<0.001$ \\
\hline EuroSCORE II & $8.78 \%$ & $7.28 \%$ & 0.161 \\
\hline NYHA I-II & $2(40)$ & $13(41.9)$ & 1 \\
\hline NYHA III-IV & $3(60)$ & $17(54.8)$ & 0.3 \\
\hline Chronic renal failure & $3(60)$ & $9(29)$ & 1 \\
\hline Atrial fibrillation/flutter & $2(40)$ & $10(32.3)$ & 0.047 \\
\hline CAD & $4(90)$ & $9(29)$ & 0.24 \\
\hline DM & $2(40)$ & $5(16.1)$ & 0.04 \\
\hline Previous CABG & $3(60)$ & $4(12.9)$ & 1 \\
\hline Previous prosthesis stenosis & $4(90)$ & $23(74.2)$ & 0.034 \\
\hline Previous prosthesis insufficiency & $1(20)$ & $23(74.2)$ & 1 \\
\hline
\end{tabular}

$C A B G=$ coronary artery bypass grafting; $C A D=$ coronary artery disease; $D M=$ diabetes mellitus; $N Y H A=N e w$ York Heart Association

immediate postoperative period as well, the nadir occurred at day 3 and platelet levels returned to baseline by day 7 . The drop in platelet count represented $47.73 \%$ from baseline, and the difference between both groups was not statistically significant until the $7^{\text {th }}$ postoperative day. However, by the $10^{\text {th }}$ day, conventional redo patients had a higher platelet counts than ViV, with statistical significance. Only one (2\%) patient in the conventional surgery group did not experience postoperative thrombocytopenia.

Differences between conventional and transcatheter groups were more pronounced in aortic patients. Conventional surgery patients presented thrombocytopenia in the immediate postoperative period and the nadir was sooner, on the $3^{\text {rd }}$ postoperative day. The curves crossed and recovery of platelet count was faster in the conventional group, reaching normal preoperative levels by the $6^{\text {th }}$ day and presenting a considerable difference by the $10^{\text {th }}$ postoperative day.

The nadir of the curve was on the $4^{\text {th }}$ day and represented a drop of $52.4 \%$ in platelet count.

From the end of the fist week until the $10^{\text {th }}$ day, the highest level of thrombocytopenia in the ViV group was statistically significant, as shown in Figure 3. In the aortic control group, only $3(7.5 \%)$ patients remained with normal platelet counts in the postoperative period.

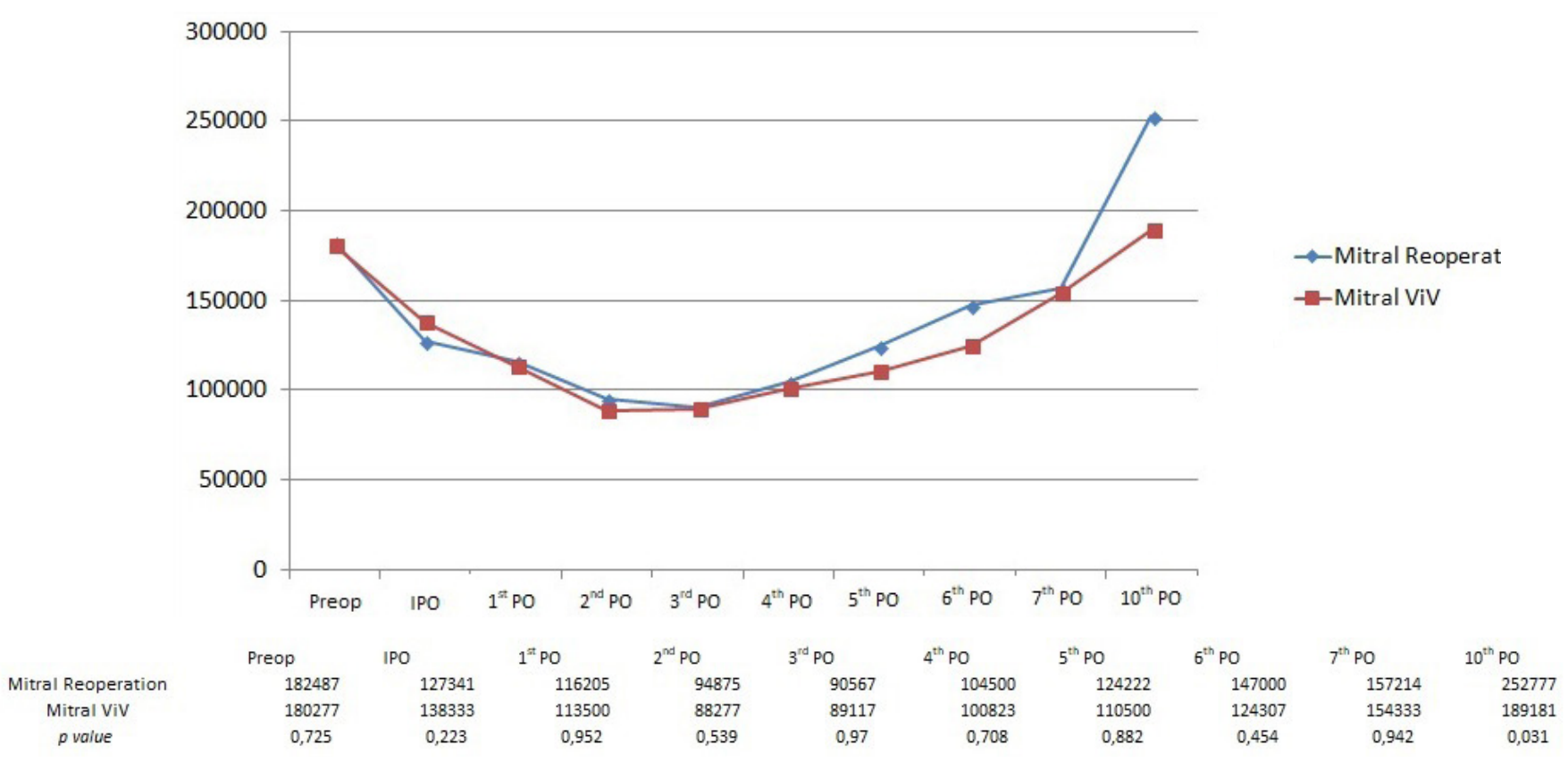

Fig. 2 - Comparison between mean platelet values for conventional mitral reoperation and mitral valve-in-valve (VIV) patients. 


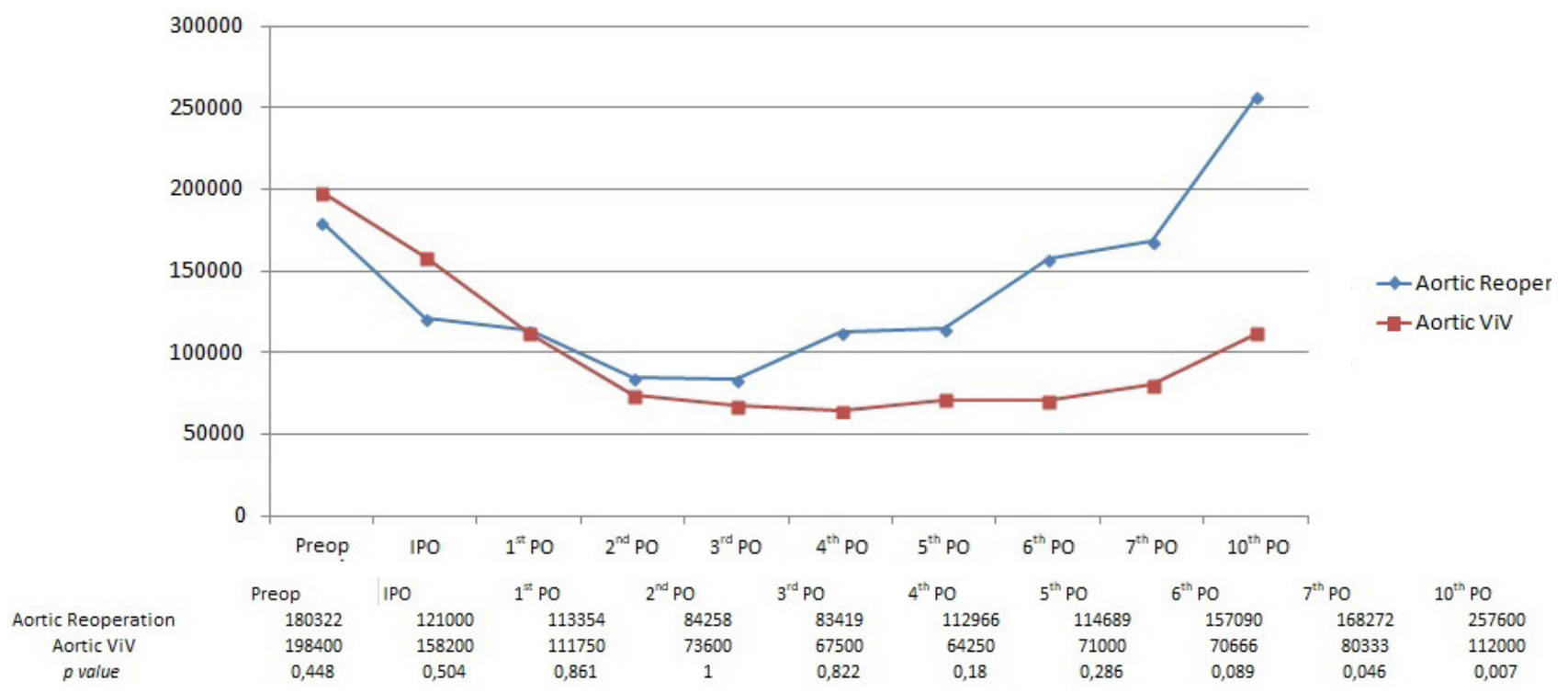

Fig. 3 - Comparison between mean platelet values for conventional aortic reoperation and aortic valve-in-valve (VIV) patients.

\section{Predictors and Outcomes}

To further refine the data analysis, we divided all ViV patients considering the degree of thrombocytopenia presented, according to each individual's initial platelet count (drop $\geq 50 \%$ or $<50 \%$ ), instead of accounting only for the absolute lowest platelet value. This is shown in Table 4, where 16 (57\%) patients presented with a drop in platelets $\geq 50 \%$ and 12 (43\%) had less than a $50 \%$ drop.

There were also no statistically significant differences between patient's characteristics in both groups, except for the preoperative platelet count. A normal platelet count from baseline was significantly correlated with a greater fall in platelet levels, over 50\%. Paradoxically, patients who already had mild to moderate preoperative thrombocytopenia experienced a less pronounced drop in platelet count. Nevertheless, a drop over $50 \%$ was not correlated with higher risk of mortality, major bleeding, acute renal failure, or other studied outcomes.

\section{DISCUSSION}

In this retrospective study, we aimed to correlate pre- and postoperative characteristics of ViV patients with postoperative thrombocytopenia, which occurred in all of our patients. There were expected preoperative differences between patients in the ViV group and in the conventional reoperation group. Naturally, those who underwent ViV procedures had been deemed as high/ extremely high risk for conventional surgery by our heart team, and therefore were older, with more comorbidities, a higher incidence of chronic renal failure and previous CABG. Nevertheless, the EuroSCORE II values, although higher for the ViV group, did not show a statistically significant difference between both groups.

Surprisingly, although thrombocytopenia is regarded as multifactorial, it has, for a long time, been attributed mainly to the installation of CPB, since upon initiation of bypass platelets drop to approximately 50\% from baseline ${ }^{[12]}$. The unavoidable hemodilution that succeeds, the sheer stress to the platelets, by roller or centrifugal pumps, and extracorporeal plastic tubing interaction, have been commonly charged with causing their ultimate destruction ${ }^{[12,19]}$. Other well-established factors associated with thrombocytopenia in cardiac surgery are the use of intra-aortic balloon pumps (IABP), sepsis and drugrelated, mainly heparin-induced thrombocytopenia $(\mathrm{HIT})$, a rare phenomenon with incidence around 1\%[11-13].

However, thrombocytopenia occurred in a similar fashion in patients submitted to transcatheter ViV implants, which were not placed in CPB. Platelet curves for conventional surgery and mitral ViV implants were particularly similar, with no statistical difference until the $7^{\text {th }}$ postoperative day, whereas curves comparing procedures for the aortic position showed significant difference, with a more pronounced and prolonged platelet drop in ViV implants.

Therefore, we may infer that, although multifactorial, CPB may not necessarily be the main cause of thrombocytopenia after valvular heart surgery, and platelet interaction with the bioprosthetic valve components such as bovine pericardium, Teflon felts, chromium-cobalt or nitinol stent frames, may play a much more important role. This could explain why aortic ViV patients experienced a higher degree of thrombocytopenia in comparison to mitral ViV.

Physiologically, transvalvular pressure and velocity gradients are higher in aortic than in mitral position, which could justify higher stress levels to platelets and translate into greater platelet degradation and consumption.

To the authors' knowledge, this is the first study to try to correlate the degree of thrombocytopenia after ViV procedures and the incidence of adverse events. Jilaihawi et al. ${ }^{[1]]}$ conducted a similar study regarding thrombocytopenia, comparing 246 TAVR patients (200 transfemoral and 46 transapical) with 57 high-risk surgical aortic valve replacement (SAVR) patients. They found very similar platelet curves between both groups, with a 
Table 4. Baseline and postoperative characteristics of valve-in-valve patients according to the degree of thrombocytopenia in relation to each individual's baseline platelet value and proportional drop in platelet count.

\begin{tabular}{|c|c|c|c|}
\hline & Drop $<50 \%(n=12)$ & Drop $>50 \%(n=16)$ & $P$-value \\
\hline \multicolumn{4}{|l|}{ Preoperative } \\
\hline Age & 54 & 65 & 0.205 \\
\hline EuroSCORE II & $10(2-33)$ & $9(3-26)$ & 0.478 \\
\hline NYHA I-II & $4(33.3)$ & $5(31.25)$ & 0.612 \\
\hline NYHA III-IV & $8(66.6)$ & $11(68.75)$ & 0.612 \\
\hline Chronic renal failure & $6(50)$ & $9(56.25)$ & 0.521 \\
\hline Atrial fibrillation/flutter & $8(66.6)$ & $9(56.25)$ & 0.435 \\
\hline CAD & $3(25)$ & $7(43.75)$ & 0.268 \\
\hline DM & $1(8.3)$ & $6(37,5)$ & 0.091 \\
\hline CABG & $3(25)$ & $4(25)$ & 0.666 \\
\hline Previous prosthesis stenosis & $6(50)$ & $6(37.5)$ & 0.391 \\
\hline Previous prosthesis insufficiency & $6(50)$ & $10(62.5)$ & 0.391 \\
\hline Absent or mild paravalvular leak & $11(91.6)$ & $15(93.75)$ & 0.389 \\
\hline Moderate paravalvular leak & $1(8.3)$ & $1(6.25)$ & 0.389 \\
\hline No preoperative thrombocytopenia & $3(25)$ & $13(81.25)$ & 0.004 \\
\hline Mild preoperative thrombocytopenia & $6(50)$ & $2(12.5)$ & 0.04 \\
\hline Moderate preoperative thrombocytopenia & $3(25)$ & $1(6.25)$ & 0.196 \\
\hline \multicolumn{4}{|l|}{ Postoperative } \\
\hline Absent or mild paravalvular regurgitation & $11(91.6)$ & $15(93.75)$ & 0.389 \\
\hline Moderate paravalvular regurgitation & $1(8.3)$ & $1(6.25)$ & 0.389 \\
\hline Death & $2(16.6)$ & $3(18.75)$ & 0.643 \\
\hline Major bleeding & $1(8.3)$ & $1(6.250)$ & 0.683 \\
\hline Acute renal failure & $7(58.3)$ & $10(62.5)$ & 0.565 \\
\hline Sepsis & $5(41.6)$ & $6(37.5)$ & 0.565 \\
\hline
\end{tabular}

$\mathrm{CABG}=$ coronary artery bypass grafting; $\mathrm{CAD}=$ coronary artery disease; $\mathrm{DM}=$ diabetes mellitus; NYHA=New York Heart Association

greater decrease in the SAVR group, unlike our ViV aortic group comparison. Major thrombocytopenia occurred in over one third of patients in the TAVR group, which proved to be benign and self-limited in most cases. As in our study, they were unable to find a significant relationship between moderate/severe thrombocytopenia and adverse events, when platelet count normalizes before the end of the first postoperative month. However, in the rare cases in which thrombocytopenia was found to be persistent (lasting longer than $30 \pm 10$ days), this was an independent predictor of mortality (HR 3,27).

In a comparable fashion, Dvir et al. ${ }^{[14]}$ conducted a study with 488 TAVR patients seeking an association between acquired thrombocytopenia and clinical outcomes. Postoperative thrombocytopenia was extremely common and transient, with $90.2 \%$ of patients presenting with no or mild thrombocytopenia at discharge, but $36.1 \%$ showed significant thrombocytopenia.

Interestingly, they found a nadir of platelet count $<50.000 /$ $\mu \mathrm{L}$ to be a highly specific marker for 30-day mortality. The occurrence of severe thrombocytopenia, analyzed through multivariate logistic regression, proved to be and independent risk factor for one-year mortality $(\mathrm{OR} 3,44)$.

The authors were able to correlate the degree of thrombocytopenia with the following adverse events at 30 days: cardiovascular death, sepsis, major bleeding, greater units of blood transfusion, acute kidney injury (AKI) and prolonged ICU stay. Therefore, they propose that thrombocytopenia can be used as an excellent prognostic marker for adverse short- and long-term outcomes after TAVR.

In our study population, there was no significant correlation between the degree of thrombocytopenia and the incidence of adverse events, and no preoperative or immediate postoperative factors could predict the occurrence of thrombocytopenia. Jilaihawi et al. ${ }^{[11]}$ found significant association between moderate/ severe persistent thrombocytopenia and hepatic cirrhosis, male sex and preoperative thrombocytopenia. Dvir et al. ${ }^{[14]}$, on the other hand, found the following characteristics associated with severe 
thrombocytopenia after TAVR: preoperative thrombocytopenia, leukopenia, vascular access complications, major bleeding, multiple transfusions, AKI, and sepsis. In contrast, our ViV group analysis showed that patients with normal preoperative platelet counts were at a higher risk of presenting with a greater drop in platelet counts $>50 \%$ than patients who already presented with mild or even moderate thrombocytopenia.

Two other studies focused on thrombocytopenia after TAVR were conducted by Flaherty et al. ${ }^{[20]}$, who analyzed 90 TAVR patients who received the Edwards SAPIEN valve; and McCabe et al. [21], which compared 112 TAVR patients with 105 balloon aortic valvuloplasties (BAV) patients. Flaherty et al.[20] identified that thrombocytopenia occurred in 79\% of post-TAVR patients, and independent preoperative predictors for moderate/severe thrombocytopenia were baseline thrombocytopenia, leaner body mass, smaller aortic valve area, higher peak aortic jet velocity and worse renal function. The occurrence of major thrombocytopenia was a predictor for major vascular complications and major bleeding. The study conducted by McCabe et al.[21], on the other hand, found that, although there was a significant difference in the prevalence of thrombocytopenia in TAVR patients (69\%) in comparison to BAV (37\%), this did not translate into any major clinical outcomes, exceptfor an increased use of hemocomponents. The possibility of severe or persistent thrombocytopenia following TAVR remains a major concern, since bleeding following TAVR is independently associated with increased mortality.

Furthermore, there may be more on the issue of thrombocytopenia than meets the eye. A Polish study, conducted by Mitrosz et al.[22], analyzed 32 TAVR patients and found that the only factor which positively correlated with drop platelets was the amount of iodinated contrast used during the procedure. This aspect was not taken into consideration in any of the other studies mentioned above.

Interestingly, in all TAVR studies, there was a considerable number of patients who did not present thrombocytopenia after the procedure. However, in our ViV study, all patients developed postoperative thrombocytopenia. These were all high-risk patients, with similar baseline characteristics, whose differences can be credited mainly to the surgical procedure itself (ViV rather than TAVR) and to the type of valve prosthesis utilized (Braile INOVARE, Medtronic COREVALVE and Edwards SAPIEN). Considering differences in surgical bioprosthetic valves as an example, Piccardo et al.[23] have already shown a higher incidence of thrombocytopenia after surgical implant of the Freedom Solo valve in comparison to Carpentier-Edwards Perimount (25\% vs. $3 \%$, respectively), which did not translate into deleterious events.

\section{CONCLUSION}

Thrombocytopenia after ViV procedures remains an extremely frequent finding, with unknown origins. In our study population, this phenomenon occurred in all patients; however, the degree of thrombocytopenia could not be associated with the incidence of adverse outcomes, probably due to a limited number of patients. Thrombocytopenia, therefore, did not prove itself to be a marker of worse prognosis in our ViV population.

Since previous studies, with larger series of patients, have shown positive implications of the decrease in platelet counts and higher postoperative risk in TAVR patients, we believe that further investigation of this matter in the ViV cohort should be encouraged.

\section{Authors' roles \& responsibilities}

RCS Substantial contributions to the conception or design of the work; or the acquisition, analysis, or interpretation of data for the work; drafting the work or revising it critically for important intel-lectual content; final approval of the version to be published

LP Substantial contributions to the conception or design of the work; or the acquisition, analysis, or interpretation of data for the work; drafting the work or revising it critically for important intellec-tual content; final approval of the version to be published

GV Substantial contributions to the conception or design of the work; or the acquisition, analysis, or interpretation of data for the work; drafting the work or revising it critically for important intellec-tual content; final approval of the version to be published

JA Agreement to be accountable for all aspects of the work in ensuring that questions related to the accuracy or integrity of any part of the work are appropriately investigated and resolved; final ap-proval of the version to be published

LLA Substantial contributions to the conception or design of the work; or the acquisition, analysis, or interpretation of data for the work; final approval of the version to be published

HR Substantial contributions to the conception or design of the work; or the acquisition, analysis, or interpretation of data for the work; final approval of the version to be published

ROS Substantial contributions to the conception or design of the work; or the acquisition, analysis, or interpretation of data for the work; final approval of the version to be published

FT Drafting the work or revising it critically for important intellectual content; final approval of the version to be published

PMAP Drafting the work or revising it critically for important intellectual content; final approval of the version to be published

JHP Drafting the work or revising it critically for important intellectual content; final approval of the version to be published

FBJ Final approval of the version to be published

\section{REFERENCES}

1. Wang HL, Aguilera C, Knopf KB, Chen TM, Maslove DM, Kuschner WG. Thrombocytopenia in the intensive care unit. J Intensive Care Med. 2013;28(5):268-80

2. Vanderschueren $S$, De Weerdt A, Malbrain M, Vankersschaever D, Frans E, Wilmer A, et al. Thrombocytopenia and prognosis in intensive care. Crit Care Med. 2000;28(6):1871-6. 
3. Marshall JC, Cook DJ, Christou NV, Bernard GR, Sprung CL, Sibbald WJ. Multiple organ dysfunction score: a reliable descriptor of a complex clinical outcome. Crit Care Med. 1995;23(10):1638-52.

4. Strand K, Flaatten H. Severity scoring in the ICU: a review. Acta Anaesthesiol Scand. 2008;52(4):467-78.

5. Ferreira FL, Bota DP, Bross A, Mélot C, Vincent JL. Serial evaluation of the SOFA score to predict outcome in critically ill patients. JAMA. 2001;286(14):1754-8

6. Le Gall JR, Klar J, Lemeshow S, Saulnier F, Alberti C, Artigas A, et al. The Logistic Organ Dysfunction system. A new way to assess organ dysfunction in the intensive care unit. ICU Scoring Group. JAMA. 1996;276(10):802-10

7. Rapsang AG, Shyam DC. Scoring systems in the intensive care unit: a compendium. Indian J Crit Care Med. 2014;18(4):220-8.

8. Crowther MA, Cook DJ, Meade MO, Griffith LE, Guyatt GH, Arnold DM, et al. Thrombocytopenia in medical-surgical critically ill patients: prevalence, incidence, and risk factors. J Crit Care. 2005;20(4):348-53.

9. Drews RE, Weinberger SE. Thrombocytopenic disorders in critically ill patients. Am J Respir Crit Care Med. 2000;162(2 Pt 1):347-51.

10. Rezende E, Morais G, Silva Junior JM, Oliveira AM, Souza JM, Toledo DO, et al. Thrombocytopenia in cardiac surgery: diagnostic and prognostic importance. Rev Bras Cir Cardiovasc. 2011;26(1):47-53.

11. Jilaihawi H, Doctor N, Chakravarty T, Kashif M, Mirocha J, Cheng W, et al. Major thrombocytopenia after balloon-expandable transcatheter aortic valve replacement: prognostic implications and comparison to surgical aortic valve replacement. Catheter Cardiovasc Interv. 2015;85(1):130-7.

12. Matthai WH Jr. Thrombocytopenia in cardiovascular patients: diagnosis and management. Chest. 2005;127(2 Suppl):46S-52S.

13. Steele J, Kadosh B, Gulkarov IM, Salemi A. Heparin induced thrombocytopenia and cardiac surgery: a comprehensive review. J Blood Disord Transfus, 2011 S2:003.

14. Dvir D, Généreux P, Barbash IM, Kodali S, Ben-Dor I, Williams $M$, et al. Acquired thrombocytopenia after transcatheter aortic valve replacement: clinical correlates and association with outcomes. Eur Heart J. 2014;35(38):2663-71.

15. Nishimura RA, Otto CM, Bonow RO, Carabello BA, Erwin JP $3^{\text {rd }}$, Fleisher
LA, et al. 2017 AHA/ACC Focused Update of the 2014 AHA/ACC Guideline for the management of patients with valvular heart disease. A Report of the American College of Cardiology/American Heart Association Task Force on Clinical Practice Guidelines. Circulation. 2017;135(25):e1159-95.

16. Gaia DF, Couto A, Breda JR, Ferreira CB, Macedo MT, Gimenes MV, et al. Transcatheter aortic valve-in-valve implantation: a selection change? Rev Bras Cir Cardiovasc. 2012;27(3):355-61.

17. Gaia DF, Breda JR, Duarte Ferreira CB, Marcondes de Souza JA, Macedo MT, Gimenes MV, et al. New Braile Inovare transcatheter aortic prosthesis: clinical results and follow-up. Eurolntervention. 2015;11(6):682-9.

18. Kappetein AP, Head SJ, Généreux P, Piazza N, van Mieghem NM, Blackstone $\mathrm{EH}$, et al. Updated standardized endpoint definitions for transcatheter aortic valve implantation: the Valve Academic Research Consortium-2 consensus document. J Thorac Cardiovasc Surg. 2013;145(1):6-23.

19. Martin JF, Daniel TD, Towbridge EA. Acute and chronic changes in platelet volume and count after cardiopulmonary bypass induced thrombocytopenia in man. Thromb Haemost. 1987;57(1):55-8.

20. Flaherty MP, Mohsen A, Moore JB $4^{\text {th }}$, Bartoli CR, Schneibel E, Rawasia W, et al. Predictors and clinical impact of pre-existing and acquired thrombocytopenia following transcatheter aortic valve replacement. Catheter Cardiovasc Interv. 2015;85(1):118-29.

21. McCabe JM, Huang PH, Riedl LA, Devireddy SR, Grondell J, Connors AC, et al. Incidence and implications of idiopathic thrombocytopenia following transcatheter aortic valve replacement with the Edwards Sapien(@) valves: a single center experience. Catheter Cardiovasc Interv. 2014;83(4):633-41.

22. Mitrosz M, Kazimierczyk R, Sobkowicz B, Waszkiewicz E, Kralisz P, Frank $M$, et al. The causes of thrombocytopenia after transcatheter aortic valve implantation. Thromb Res. 2017;156:39-44.

23. Piccardo A, Rusinaru D, Petitprez B, Marticho P, Vaida I, Tribouilloy $C$, et al. Thrombocytopenia after aortic valve replacement with freedom solo bioprosthesis: a propensity study. Ann Thorac Surg. 2010;89(5):1425-30. 